\title{
Borexino's search for low-energy neutrino and antineutrino signals correlated with gamma-ray bursts
}

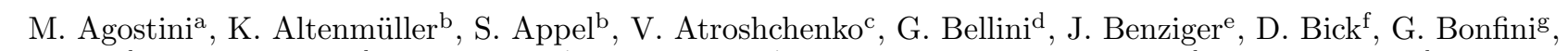
D. Bravo ${ }^{\text {h }}$, B. Caccianiga ${ }^{\mathrm{d}}$, F. Calaprice ${ }^{\mathrm{i}}$, A. Caminata ${ }^{\mathrm{j}}$, M. Carlini $^{\mathrm{g}}$, P. Cavalcantes,h, A. Chepurnov ${ }^{\mathrm{k}}$, K. Choi $^{\mathrm{l}}$,

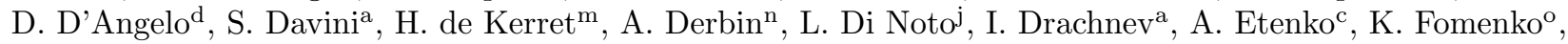
D. Franco ${ }^{\mathrm{m}}$, F. Gabrieles , C. Galbiati ${ }^{\mathrm{i}}$, C. Ghiano ${ }^{\mathrm{j}}$, M. Giammarchi ${ }^{\mathrm{d}}$, M. Goeger-Neff ${ }^{\mathrm{b}}$, A. Goretti ${ }^{\mathrm{i}}$, M. Gromov ${ }^{\mathrm{k}}$, C. Hagner ${ }^{f}$, E. Hungerford ${ }^{\mathrm{p}}$, Aldo Ianni ${ }^{\mathrm{g}}$, Andrea Ianni ${ }^{\mathrm{i}}$, A. Jany ${ }^{\mathrm{q}}$, K. Jedrzejczak ${ }^{\mathrm{q}}$, D. Jeschke ${ }^{\mathrm{b}}$, V. Kobychev ${ }^{\mathrm{r}}$, D. Korablev ${ }^{\mathrm{o}}$, G. Korga ${ }^{\mathrm{p}}$, D. Kryn ${ }^{\mathrm{m}}$, M. Laubenstein ${ }^{\mathrm{g}}$, B. Lehnert ${ }^{\mathrm{s}}$, E. Litvinovich ${ }^{\mathrm{c}, \mathrm{t}}$, F. Lombardi ${ }^{\mathrm{g}}$, P. Lombardi ${ }^{\mathrm{d}}$,

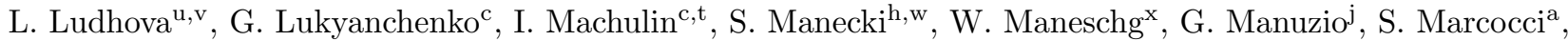

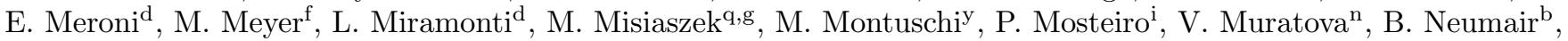

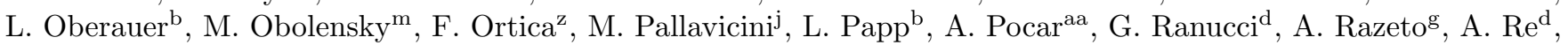
A. Romani ${ }^{\mathrm{z}}$, R. Roncin ${ }^{\mathrm{g}, \mathrm{m}}$, N. Rossi ${ }^{\mathrm{g}}$, S. Schönert ${ }^{\mathrm{b}}$, D. Semenov ${ }^{\mathrm{n}}$, M. Skorokhvatov ${ }^{\mathrm{c}, \mathrm{t}}$, O. Smirnov ${ }^{\mathrm{o}}$, A. Sotnikov ${ }^{\mathrm{o}}$,

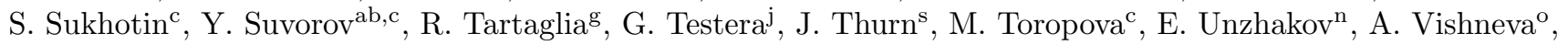

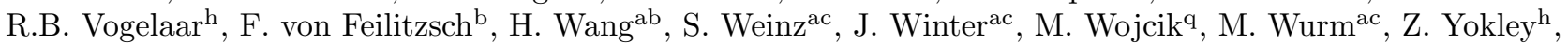
O. Zaimidoroga ${ }^{\circ}$, S. Zavatarellij ${ }^{\mathrm{j}}$, K. Zuber ${ }^{\mathrm{s}}$, G. Zuzel ${ }^{\mathrm{q}}$

The Borexino Collaboration

${ }^{a}$ Gran Sasso Science Institute (INFN), 67100 E'Aquila, Italy

${ }^{b}$ Physik-Department and Excellence Cluster Universe, Technische Universität München, 85748 Garching, Germany ${ }^{c}$ National Research Centre Kurchatov Institute, 123182 Moscow, Russia

${ }^{d}$ Dipartimento di Fisica, Università degli Studi e INFN, 20133 Milano, Italy

${ }^{e}$ Chemical Engineering Department, Princeton University, Princeton, NJ 08544, USA

${ }^{f}$ Institut für Experimentalphysik, Universität, 22761 Hamburg, Germany

${ }^{g}$ INFN Laboratori Nazionali del Gran Sasso, 67010 Assergi (AQ), Italy

${ }^{h}$ Physics Department, Virginia Polytechnic Institute and State University, Blacksburg, VA 24061, USA

${ }^{i}$ Physics Department, Princeton University, Princeton, NJ 08544, USA

${ }^{j}$ Dipartimento di Fisica, Università degli Studi e INFN, Genova 16146, Italy

${ }^{k}$ Lomonosov Moscow State University Skobeltsyn Institute of Nuclear Physics, 119234 Moscow, Russia

${ }^{l}$ Department of Physics and Astronomy, University of Hawaii, Honolulu, HI 96822, USA

${ }^{m}$ AstroParticule et Cosmologie, Université Paris Diderot, CNRS/IN2P3, CEA/IRFU, Observatoire de Paris, Sorbonne Paris Cité, 75205 Paris Cedex 13, France

${ }^{n}$ St. Petersburg Nuclear Physics Institute NRC Kurchatov Institute, 188350 Gatchina, Russia

${ }^{o}$ Joint Institute for Nuclear Research, 141980 Dubna, Russia

${ }^{p}$ Department of Physics, University of Houston, Houston, TX 77204, USA

${ }^{q}$ M. Smoluchowski Institute of Physics, Jagiellonian University, 30059 Krakow, Poland ${ }^{r}$ Institute for Nuclear Research, $03680 \mathrm{Kiev}$, Ukraine

${ }^{s}$ Department of Physics, Technische Universität Dresden, 01062 Dresden, Germany

${ }^{t}$ National Research Nuclear University MEPhI (Moscow Engineering Physics Institute), 115409 Moscow, Russia

${ }^{u} I K P$-2 Forschungzentrum Jülich, 52428 Jülich, Germany

${ }^{v}$ RWTH Aachen University, 52062 Aachen, Germany

${ }^{w}$ Physics Department, Queen's University, Kingston ON K7L 3N6, Canada

${ }^{x}$ Max-Planck-Institut für Kernphysik, 69117 Heidelberg, Germany

${ }^{y}$ Dipartimento di Fisica e Scienze della Terra Università degli Studi di Ferrara e INFN, Via Saragat 1-44122, Ferrara, Italy

${ }^{z}$ Dipartimento di Chimica, Biologia e Biotecnologie, Università e INFN, 06123 Perugia, Italy

${ }^{a}$ Amherst Center for Fundamental Interactions and Physics Department, University of Massachusetts, Amherst, MA 01003, $U S A$

${ }^{a b}$ Physics and Astronomy Department, University of California Los Angeles (UCLA), Los Angeles, California 90095, USA

${ }^{a c}$ Institute of Physics and Excellence Cluster PRISMA, Johannes Gutenberg-Universität Mainz, 55099 Mainz, Germany

\section{Abstract}

A search for neutrino and antineutrino events correlated with 2,350 gamma-ray bursts (GRBs) is performed with Borexino data collected between December 2007 and November 2015. No statistically significant excess over background is observed. We look for electron antineutrinos $\left(\bar{\nu}_{e}\right)$ that inverse beta decay on protons with energies from $1.8 \mathrm{MeV}$ to $15 \mathrm{MeV}$ and set the best limit on the neutrino fluence from GRBs below $8 \mathrm{MeV}$. The signals from neutrinos and antineutrinos from GRBs that scatter on electrons are also searched for, a detection channel made possible by the particularly radio-pure scintillator of Borexino. We obtain currently the best limits on the neutrino fluence of all flavors and species below $7 \mathrm{MeV}$. Finally, time correlations between GRBs and bursts of events are investigated. Our analysis combines two semi-independent data acquisition systems for the first time: the primary Borexino readout optimized for solar neutrino physics up to a few $\mathrm{MeV}$, and a fast waveform digitizer system tuned for events above $1 \mathrm{MeV}$.

Keywords: Neutrinos, Antineutrinos, Gamma-ray bursts, low energy/MeV neutrinos 


\section{Introduction}

Gamma ray bursts (GRBs) are among the most energetic events known in the Universe, with a typical apparent energy release of $10^{54} \mathrm{erg}$ ( or $\sim 1$ solar mass), assuming isotropic emission of energy. The average rate of observed GRBs is about 1 event per day from the entire sky. The observer-frame duration of gamma ray emission in the $\mathrm{MeV}$ range can be less than $2 \mathrm{~s}$ (for a smaller sub-class of so called short GRBs) or, more typically, is of the order of 10 to 100 seconds. Longer afterglows in X-rays, optical, and radio wavelengths are also observed. The measured redshifts of optical afterglows $(z=0.01 . .8 .2)$ allow to attribute GRBs as extra-galactic events, whose progenitors lie at cosmological distances.

Currently, there is no universally accepted model of GRBs. However, the long GRBs are usually linked to the rotating cores of very massive stars collapsed into neutron stars (NS) or black holes (BH) 1, 2]. Short GRBs can result from binary mergers of $\mathrm{NS}+\mathrm{NS}$ or $\mathrm{NS}+\mathrm{BH}[2]$. These models usually assume that neutrino cooling dominates over the electromagnetic one with neutrino energies in the $\mathrm{MeV}$ range [3]. In these models, the energy emitted in the form of $\mathrm{MeV}$ thermal neutrinos is of the order of one Solar mass $\left(M_{\odot} c^{2} \approx 2 \cdot 10^{54}\right.$ ergs $)$ while the energy released in gamma quanta is up to $\sim 100$ times less [4, 5]. Even for the nearest GRBs with $z \sim 0.01$ (for the standard $\Lambda$ CDM cosmological model [6], this comoving distance is $\sim 10^{26} \mathrm{~cm}$ or $30 \mathrm{Mpc}$ ), the expected fluence of low-energy neutrinos at Earth is equal to $10^{5}-10^{6} \mathrm{~cm}^{-2}$, which is too small to be observed by current detectors. However, there exist other models for the origin of GRBs, such as cusps of superconducting cosmic strings [7] which can better explain some peculiarities of GRBs [8]. Some of these models are predicting the fluence of $\mathrm{MeV}$-range neutrinos to be up to $10^{10}$ times larger than that of gamma quanta. The neutrino fluence in these models can be estimated as [4]:

$$
\begin{aligned}
\Phi_{\nu} & =10^{8} \mathrm{~cm}^{-2}\left(\frac{\eta_{\gamma}}{10^{-10}}\right)^{-1}\left(\frac{E_{\nu}}{100 \mathrm{MeV}}\right)^{-1} \\
& \times\left(\frac{F_{\gamma}}{10^{-6} \mathrm{erg} \cdot \mathrm{cm}^{-2}}\right)
\end{aligned}
$$

where $\eta_{\gamma}$ is the ratio of photon and neutrino fluences, $F_{\gamma}$ is the observed gamma ray energy fluence of the GRB. Thus, for a typical GRB with gamma energy release of $3 \times 10^{51} \mathrm{erg}$ at redshift $z=2\left(F_{\gamma}=10^{-6} \mathrm{erg} \cdot \mathrm{cm}^{-2}\right)$, the predicted fluence of neutrinos with energy of $\sim 10 \mathrm{MeV}$ is $\sim 10^{8}-10^{9} \mathrm{~cm}^{-2}$. For comparison, the observed flux of solar neutrinos is about $6 \cdot 10^{10} \mathrm{~cm}^{-2} \cdot \mathrm{s}^{-1}$, of geoantineutrinos is about $5 \cdot 10^{6} \mathrm{~cm}^{-2} \cdot \mathrm{s}^{-1} 9$. It demonstrates that the sensitivity level of existing neutrino detectors in the $\mathrm{MeV}$ range is close to the fluxes expected in several GRB models, if one uses a big set of GRBs. Hereinafter, the energy of neutrino refers to the observed energy; the emitted energy has to be multiplied by factor $1+z$ which is not important due to large uncertainties in models predictions.

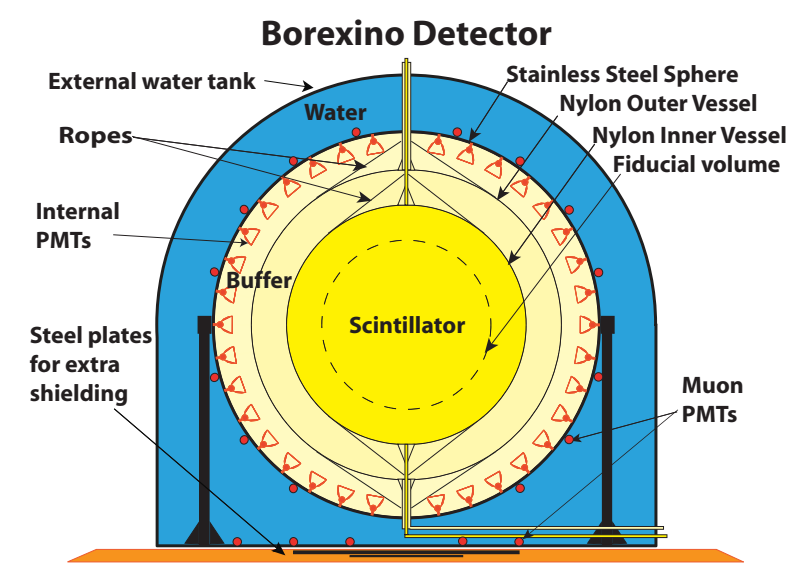

Figure 1: Schematic view of the Borexino detector.

Production of $\mathrm{TeV}$ and $\mathrm{PeV}$ neutrinos by protons accelerated by the plasma shock wave of GRBs was discussed 10, 11] and such high-energy neutrinos were searched for by AMANDA [12, ANITA [13, ANTARES [14, 15, Baikal [16], IceCube [17, 18, and SuperKamiokande [19], but no signal was found. The searches for GRB neutrinos in the $\mathrm{MeV}$ energy range have been performed by four experiments: SuperKamiokande [19], SNO [20, KamLAND 21, and BUST [22].

The SuperKamiokande collaboration searched for electron and muon neutrinos and antineutrinos in the energy range of $7-80 \mathrm{MeV}$. The SNO collaboration searched for electron neutrinos, electron antineutrinos, and for (anti)neutrinos of non-electron flavors in the range of 5$13 \mathrm{MeV}$. The KamLAND collaboration set upper limits on electron antineutrino fluence associated with GRBs with known redshift, in the energy ranges of 7.5-100 MeV and (after the Japanese nuclear reactors were switched off in 2011) of 1.8-100 MeV. The BUST (Baksan Underground Scintillation Telescope) was sensitive to electron neutrinos and antineutrinos in the energy interval of $20-100 \mathrm{MeV}$. None of these four experiments found any correlation between GRBs and neutrino events in their detectors.

In this paper, we present a search for possible correlations between GRBs and (anti)neutrino events for all neutrino flavours in the Borexino detector.

\section{The Borexino detector}

Borexino is a liquid scintillator detector placed in the Hall C of the underground Laboratory Nazionali del Gran Sasso (LNGS) in central Italy. The radiopurity of this unsegmented detector has reached unprecedented levels through the techniques described in 23. This was fundamental in reaching the primary goal of Borexino, the real time spectroscopy of solar neutrinos below $1 \mathrm{MeV}$ [24, 25]. The detector design 26 is based on the principle of graded shielding, with the inner scintillator core at the center of a 
set of concentric shells of decreasing radio-purity from inside to outside (see Fig. 1). The active medium, 278 tons of pseudocumene (PC) doped with $1.5 \mathrm{~g} / \mathrm{l}$ of PPO $(2,5-$ diphenyloxazole, a fluorescent dye), is confined within a thin spherical nylon vessel with a radius of $4.25 \mathrm{~m}$ surrounded by 2212 8" (ETL 9351) photomultipliers (PMTs), defining the so called Inner Detector (ID). All but 371 PMTs are equipped with aluminum light concentrators designed to increase the light collection efficiency. The detector core is shielded from external radiation by 890 tons of buffer liquid, a solution of PC and 3-5 g/l of the light quencher dimethylphthalate (DMP). The buffer is divided in two volumes by the second nylon vessel with a $5.75 \mathrm{~m}$ radius, preventing inward radon diffusion. A $6.85 \mathrm{~m}$ radius Stainless Steel Sphere (SSS) encloses the central part of the detector and serves as a support for PMTs. An external domed water tank of $9 \mathrm{~m}$ radius and $16.9 \mathrm{~m}$ height, filled with ultra-high purity water, serves as a passive shield against neutrons and gamma rays as well as an active muon veto. The Cherenkov light radiated by muons passing through the water is measured by 208 8" external PMTs defining the so called Outer Detector (OD) [27.

Several calibration campaigns with radioactive sources 28] allowed us to decrease the systematic errors of our measurements and to optimize Geant 4 based Monte Carlo (MC) simulation. A typical energy deposit of $1 \mathrm{MeV}$ produces a signal of about 500 photoelectrons, resulting in an energy resolution of $5 \% / \sqrt{E / M e V}$. A more detailed description of the detector response can be found in 24].

The primary electronics of Borexino, in which all 2212 channels are read individually, is optimized for energies up to few MeV. For higher energies with a threshold of $1 \mathrm{MeV}$, the system of 96 fast waveform digitizers (CAEN v896, 8 bit, thereafter FADC - Flash ADC) was developed, each of them reading-in the signal summed from up to 24 PMTs, with the sampling rate of $400 \mathrm{MHz}$. The details of both systems can be found in 26. In 2009 FADC data acquisition (DAQ) system was upgraded. Starting from December 2009, it acquires data in a new hardware configuration, having a separate trigger. FADC energy scale was calibrated using the $2.22 \mathrm{MeV}$ gamma peak originating from cosmogenic neutron captures on protons, as well as by fitting the spectrum of cosmogenic ${ }^{12} \mathrm{~B}$ and Michel electrons from muons decaying inside the detector. The energy resolution of the FADC system was found to be $10 \% / \sqrt{E / \mathrm{MeV}}$. In this work, the first detailed cross-check and a combined analysis based on both systems have been performed. Advantages of each system, such as higher energy resolution in the primary DAQ or advanced software algorithms for muon and electronics-noise tagging for energies above $1 \mathrm{MeV}$ in the FADC system, were exploited together.

\section{GRB and time window selection}

We have used the GRB database compiled by the IceCube collaboration 29, which considers the data from several satellites such as SWIFT, Fermi, INTEGRAL, AGILE, Suzaki, and Konus/WIND. This database contains information about GRB's position, time of the detection, duration, energy spectrum, intensity, and, when available, the redshift. We underline that the latter is available only for about $10 \%$ of GRBs. In the period of interest from December 2007 (December 2009) to November 2015, 2350 (1813) GRBs have been observed.

Considering an upper limit of $0.23 \mathrm{eV}$ for the sum of neutrino masses [6], the maximal mass of three neutrino mass states consistent with the measured oscillation mass differences is $87 \mathrm{meV}$. We estimate the time delay, which would be induced in the observation of gamma rays and $87 \mathrm{meV}$ neutrinos emitted simultaneously. For a typical GRB redshift of $z \sim 2$, this time delay reaches $800 \mathrm{~s}$ and then shortly saturates to about $900 \mathrm{~s}$ for higher $z$ 's. The largest observed GRB redshift is $z=8.2$ for GRB 131202A.

Conservatively, we have considered a coincidence time window $\Delta t_{\text {SIG }}= \pm 1000 \mathrm{~s}$ around (before and after) the GRB detection time, in the analysis based on the neutrinoelectron elastic scattering channel. The selected time window covers the possible delay of neutrino signal which propagates slower than photons, as well as the possible earlier emission of neutrinos due to poorly constrained details of GRB physics; the reasonable assumption is that the neutrino burst and the photon burst are not separated by a time much more than the duration of the GRB, which is almost always less than $1000 \mathrm{~s}$. We excluded from this analysis those GRBs, for which the fraction of detector's up time covering $\pm 2 \Delta t_{\text {SIG }}$ (for reasons discussed below) was less than $95 \%$. The rate of events selected as Inverse Beta Decay (IBD) candidates or bursts is much lower with respect to the rate of single point-like events ( $\mathrm{sin}$ gles). This allowed us, for these two analyses, to extend the $\Delta t_{\text {SIG }}$ window to \pm 5000 s (requiring $80 \%$ detector's up time) for a less model dependent search. In the analyses where the muon veto was applied (Sec.4.1), the $\Delta t_{\text {SIG }}$ window in which the candidates (singles, IBDs, bursts) were searched for, was consequently reduced by $\sim 10 \%(\sim 4300$ muons/day passing through the ID). In the consideration of the detector's up time coverage of the $\pm(2) \Delta t_{\text {SIG }}$ windows, the muon veto was not considered. The summary of periods and the number of GRBs used in different analyses are given in Tab. 1 .

\section{Analysis and results}

We have analyzed Borexino data acquired between December 2007 and November 2015. The two semiindependent DAQ systems used in this analysis, the primary and the FADC one, have separate triggers and can operate individually, independently from each other. Different analysis approaches, as it will be specified below, 
Table 1: Summary of periods and the number of GRBs used in different analyses. The livetimes indicate up-times of different DAQ systems in a given period.

\begin{tabular}{|l|c|c|c|}
\hline DAQ system & Primary & FADC & Primary + FADC \\
\hline \hline Period & Dec 2007 - Nov 2015 & Dec 2009 - Nov 2015 & Dec 2009 - Nov 2015 \\
Observed GRBs & 2350 & 1813 & 1813 \\
\hline \hline Livetime [days] & 2302.0 & 1388.1 & 1279.7 \\
\hline Used GRBs & 1791 & 1114 & 980 \\
\hline Analysis & IBD (Sec. 4.2) \& Bursts of events (Sec. 4.4) & IBD (Sec. 4.2 & Elastic scattering (Sec. 4.3) \\
\hline
\end{tabular}

have used data from the two systems in different ways: either individually or in a combined way. In some cases the presence of the data from both systems was required, in others the FADC system was used in addition to the primary DAQ system only when available. The total live time of the primary Borexino DAQ system in this period was 2302.0 days. The FADC system in its new hardware configuration was activated only in December 2009 and was in operation during 1388.1 days. Both DAQ systems were working simultaneously for 1279.7 days.

We split the analysis into three independent parts, having in common the definition of muon events (Sec. 4.1). Firstly, we search for an electron antineutrino signal in correlation with GRBs, through IBD reaction with $1.8 \mathrm{MeV}$ kinematic threshold (Sec. 4.2). Secondly, we search for all (anti)neutrino flavours, including electron antineutrinos below the IBD threshold: we consider singles, thereby performing neutrino search through elastic scattering off electrons (Sec. 4.3). Finally, we look for the bursts of events in Borexino data, in correlation with GRBs (Sec. 4.4).

\subsection{Muon veto}

In all analyses, muon events have been removed. With the exception of the bursts analysis done with a dynamic $2 \mathrm{~s}$ time window (Sec. 4.4), events following every tagged (and removed) muon are excluded from the data sample by applying a $2 \mathrm{~s}$ veto after muons crossing the Inner Detector (ID), and a $2 \mathrm{~ms}$ veto for those crossing only the Outer Detector (OD). The standard Borexino muon identification [27], including both OD veto and ID pulse-shape muon tagging, is prone to mistakenly tag point-like events above few $\mathrm{MeV}$ as muons. In the previous Borexino analysis (search for antineutrinos from unknown sources 30]) extending up to about $18 \mathrm{MeV}$, we have corrected for this effect basing on MC studies. In this work, we have optimized the muon cuts for higher energies by modifying the muon tagging based on the ID pulse-shape. In particular, we have employed the anisotropy variable $S_{p}$ [24] as well as the reconstructed radius of the event instead of the variables describing the mean duration and the peak position of the light pulse. In addition, muon tagging based on the FADC waveform analysis was developed and tested against the sample of muons detected by the OD. The overefficiency to tag point-like events at energies up to few tens of $\mathrm{MeV}$ as muons was cross-checked on a sample of Michel electrons. A slightly decreased muon detection efficiency in the periods when the FADC system was not available is not of concern since in all analysis reported here we search for an excess of events in GRB correlated time windows with respect to GRB uncorrelated times.

\subsection{Inverse beta decay}

In Borexino, electron antineutrinos are detected via IBD reaction $\bar{\nu}_{e}+p \rightarrow n+e^{+}$with a threshold of $E_{\bar{\nu}}=1.8 \mathrm{MeV}$. The positron thermalizes and annihilates, giving a prompt event. The neutron thermalizes and is captured $(\tau=$ $254.5 \pm 1.8 \mu \mathrm{s})$ in most cases by a proton with emission of $2.22 \mathrm{MeV} \gamma$-ray, defining a delayed event. In about $1 \%$ of cases, neutrons are captured on ${ }^{12} \mathrm{C}$, producing $4.95 \mathrm{MeV}$ $\gamma$-rays.

The coincidences were searched for with $\Delta t=20$ (30) - $1280 \mu$ s for primary (FADC) systems, excluding muons and muon induced events. The energy of the prompt event was required to be above the value corresponding to the IBD threshold, considering the energy resolution. For the delayed event, the energy cut was tuned to cover the gammas from neutron captures both on protons and ${ }^{12} \mathrm{C}$. It was required that no event with energy above $1 \mathrm{MeV}$ would be present neither $2 \mathrm{~ms}$ before(after) the prompt(delayed) event nor between the prompt and the delayed event.

The position reconstruction is currently available only from the primary Borexino data, while it is not for FADC data. In order to cross check the consistency between both DAQ systems, we have first performed coincidence searches without application of position cuts. In the final analysis of the primary DAQ data, we have included the requirement of the mutual distance between the prompt and the delayed candidate to be $d R<1 \mathrm{~m}$. In total, the live time in the analysis of the primary(FADC) data was 2302.0(1388.1) days. After excluding GRBs with data coverage of the $\Delta t_{\text {SIG }}$ window less than $80 \%$, we have 1791(1114) GRBs left in the analysis.

Search for correlation with GRBs was performed in time window $\Delta t_{\mathrm{SIG}}= \pm 5000 \mathrm{~s}$ around each GRB. The sum of these time windows defines the integral signal time $T_{\text {SIG }}$. All identified coincidences, considering antineutrinos with energies up to $E_{\bar{\nu}_{e}}=15 \mathrm{MeV}$, were divided into $1 \mathrm{MeV}$ bins 
according to $E_{\bar{\nu}_{e}}=E_{\text {prompt }}+0.784 \mathrm{MeV}$, where $E_{\text {prompt }}$ is the energy of the prompt event. The procedure described below was then repeated for each energy bin separately.

All antineutrino-like events are divided into those detected within $T_{\mathrm{SIG}}, N_{\text {in }}$ and outside of $T_{\mathrm{SIG}}, N_{\text {out }}$. All $N_{\text {out }}$ candidates are assumed to be accidentals. The background rate $R_{\text {bgr }}$ is calculated as:

$$
R_{\mathrm{bgr}}=\frac{N_{\mathrm{out}}}{T_{\text {total }}-T_{\mathrm{SIG}}},
$$

where $T_{\text {total }}$ is the total live time of 2063.3 (1249.9) days for the primary (FADC) DAQ systems. Both $T_{\text {total }}$ and $T_{\text {SIG }}$ are after the application of the muon veto cut.

The number of background events $N_{\mathrm{bgr}}$ in $T_{\mathrm{SIG}}$ is estimated as:

$$
N_{\mathrm{bgr}}=R_{\mathrm{bgr}} T_{\mathrm{SIG}} \text {. }
$$

The $90 \%$ C.L. upper limit $\mu_{90}$ for the number of observed GRB-correlated events is calculated according to the Feldman-Cousins procedure [31] with $N_{\text {in }}$ and $N_{\text {bgr }}$ as input parameters. The upper limit $N_{90}^{I B D}$ for the number of correlated events for one GRB is then:

$$
N_{90}^{I B D}=\frac{\mu_{90}}{N_{\mathrm{GRB}}},
$$

where $N_{\text {GRB }}$ is the total number of considered GRBs.

We calculate upper limits for the fluence $\Phi_{\bar{\nu}_{e}}$ for monoenergetic antineutrinos with energy $E_{\bar{\nu}_{e}}$ :

$$
\Phi_{\bar{\nu}_{e}}\left(E_{\bar{\nu}_{e}}\right)=\frac{N_{90}^{I B D}\left(E_{\bar{\nu}_{e}}\right)}{N_{p}\langle\varepsilon\rangle \sigma\left(E_{\bar{\nu}_{e}}\right)},
$$

where $N_{90}^{I B D}\left(E_{\bar{\nu}_{e}}\right)$ is the $90 \%$ C.L. upper limit for the number of GRB-correlated events per GRB from the corresponding $1 \mathrm{MeV}$ energy bin, $N_{p}=1.6 \cdot 10^{31}$ is the number of protons in Borexino scintillator, $\langle\varepsilon\rangle$ is the average detection efficiency evaluated via MC simulations for a flat energy spectrum of antineutrinos in the range from 1.8 to $15 \mathrm{MeV}, \sigma\left(E_{\bar{\nu}_{e}}\right)$ is the IBD cross-section for antineutrino energy $E_{\bar{\nu}_{e}}$ calculated according to 32$]$.

Table 2: Borexino 90\% C.L. upper limits for fluence of electron antineutrinos from GRBs.

\begin{tabular}{|c|c|c|}
\hline $\begin{array}{c}E_{\bar{\nu}_{e}} \\
{[\mathrm{MeV}]}\end{array}$ & $\begin{array}{c}\Phi_{\bar{\nu}_{e}} \text { primary DAQ } \\
{\left[\mathrm{cm}^{-2}\right]}\end{array}$ & $\begin{array}{c}\Phi_{\bar{\nu}_{e}} \text { FADC } \\
{\left[\mathrm{cm}^{-2}\right]}\end{array}$ \\
\hline \hline 2 & $4.36 \times 10^{9}$ & $4.87 \times 10^{9}$ \\
3 & $1.13 \times 10^{8}$ & $2.64 \times 10^{8}$ \\
4 & $4.00 \times 10^{8}$ & $2.49 \times 10^{8}$ \\
6 & $3.81 \times 10^{7}$ & $5.67 \times 10^{7}$ \\
10 & $1.50 \times 10^{7}$ & $3.57 \times 10^{7}$ \\
14 & $6.96 \times 10^{6}$ & $1.04 \times 10^{7}$ \\
\hline
\end{tabular}

Results for fluence upper limits $\Phi_{\nu}$ for electron antineutrinos, obtained for each energy bin, are given in Table 2 and illustrated in Fig. 2. Results previously obtained in other experiments (SuperKamiokande [19], SNO [20], KamLAND [21]) are also shown in this plot. The BUST collaboration performed their analysis in the energy range above $20 \mathrm{MeV}[22$. Borexino limits are the strongest ones in the energy region from 2 to $8 \mathrm{MeV}$.

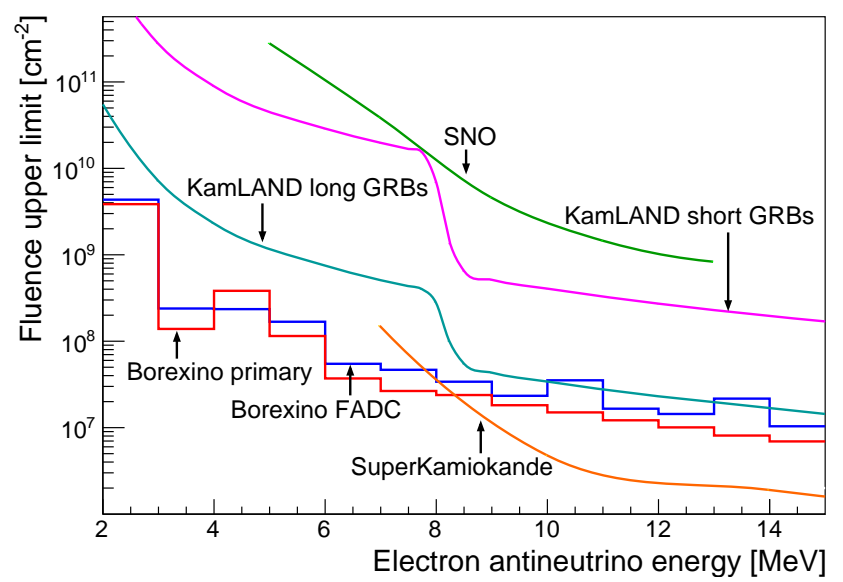

Figure 2: Fluence upper limits for electron antineutrinos from GRBs versus antineutrino energy. Borexino results are shown in comparison with results from SuperKamiokande 19], SNO [20], and KamLAND 21].

\subsection{Neutrino-electron elastic scattering}

The goal of this analysis is to search for an excess of singles in Borexino above measured background, in coincidence with GRBs in a time window $\Delta t_{\mathrm{SIG}}= \pm 1000 \mathrm{~s}$. We calculate the overall number of candidate events above $1 \mathrm{MeV}$ in $\Delta t_{\mathrm{SIG}}$, excluding muons, muon induced events, and events classified as electronic noise based on FADC pulse shape analysis.

Throughout the whole period of data-taking the counting rate of singles in Borexino is not constant. In particular, in the energy range above $1 \mathrm{MeV}$ we observe up to factor of 2 increase in rate during several scintillator purification cycles performed in 2010 - 2011. In order to take into account the effect of fluctuations in counting rate, the background was calculated as the number of singles in a $1000 \mathrm{~s}$ time windows, adjacent to $\Delta t_{\text {SIG }}$, i.e. $\Delta t_{\mathrm{BGR}}=[-2000 . .-1000 ; 1000 . .2000] \mathrm{s}$. GRBs coverage by Borexino data was then calculated for $\Delta t= \pm 2000 \mathrm{~s}$ window. Requirement of at least $95 \%$ data coverage per window leaves $N_{\mathrm{GRB}}=980$. Overall data coverage in a time window \pm 2000 s weighted over 980 remaining GRBs equals to $99.7 \%$. This analysis was performed on a data set of 1279.7 days when both the primary and the FADC systems were active.

Figure 3 shows the energy spectrum of singles measured by the primary DAQ system for the integrated time exposure $N_{\mathrm{GRB}} \times \Delta t_{\mathrm{SIG}}$ around the $980 \mathrm{GRBs}$, in the energy range from 1.5 to $15.0 \mathrm{MeV}$. The difference between this spectrum and the one measured in the time side bands, 


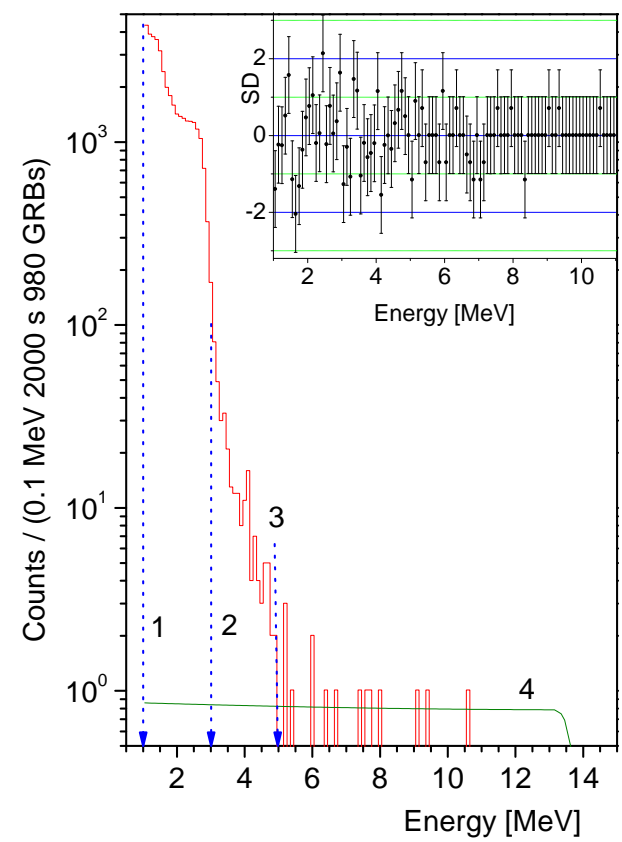

Figure 3: Borexino energy spectrum of singles in correlation with GRBs. In inset, the difference between spectra measured in $N_{\text {GRB }} \times$ $\Delta t_{\mathrm{SIG}}$ and $N_{\mathrm{GRB}} \times \Delta t_{\mathrm{BGR}}$ time windows is shown in the units of standard deviations (SD). Blue arrows indicate the three energy thresholds $T_{t h}$ from Eq. 7 chosen for the separate analysis (details in text). Line 4 shows the expected spectrum of recoil electrons for the fluence $1 \times 10^{10} \mathrm{~cm}^{-2}$ per one GRB of $14 \mathrm{MeV}$ neutrinos.

$N_{\mathrm{GRB}} \times \Delta t_{\mathrm{BGR}}$, is shown in the inset. No statistically significant excess is observed in correlations with GRBs.

The scattering of monoenergetic neutrinos with energy $E_{\nu}$ off electrons leads to recoil electrons with a Comptonlike continuous energy spectrum with maximum energy $T_{\nu}^{\max }=2 E_{\nu}^{2} /\left(m_{e}+2 E_{\nu}\right)$. Thus, the fluence limit for neutrinos of energy $E_{\nu}$ should be calculated by a modified version of Eq. 5 .

$$
\Phi_{\nu}\left(E_{\nu}\right)=\frac{N_{90}^{\nu e}\left(E_{\nu}\right)}{N_{e} \sigma_{\mathrm{eff}}\left(E_{\nu}\right)},
$$

where $N_{e}=8.8 \cdot 10^{31}$ is the number of electrons in the Borexino scintillator and $\sigma_{\text {eff }}\left(E_{\nu}\right)$ is the effective cross section for neutrinos with energy $E_{\nu}$ via the detection of recoil electrons with reconstructed energy $T$ in the interval $\left(T_{\mathrm{th}}>0, T_{\nu}^{u p}\right)$, with $T_{\nu}^{u p}=T_{\nu}^{\max }+\sigma_{T}(T)$ and $\sigma_{T}$ the energy resolution of the detector, with $100 \%$ detection efficiency and can be expressed as:

$$
\sigma_{\mathrm{eff}}\left(E_{\nu}\right)=\int_{T_{t h}}^{T_{\nu}^{u p}} F(T) d T
$$

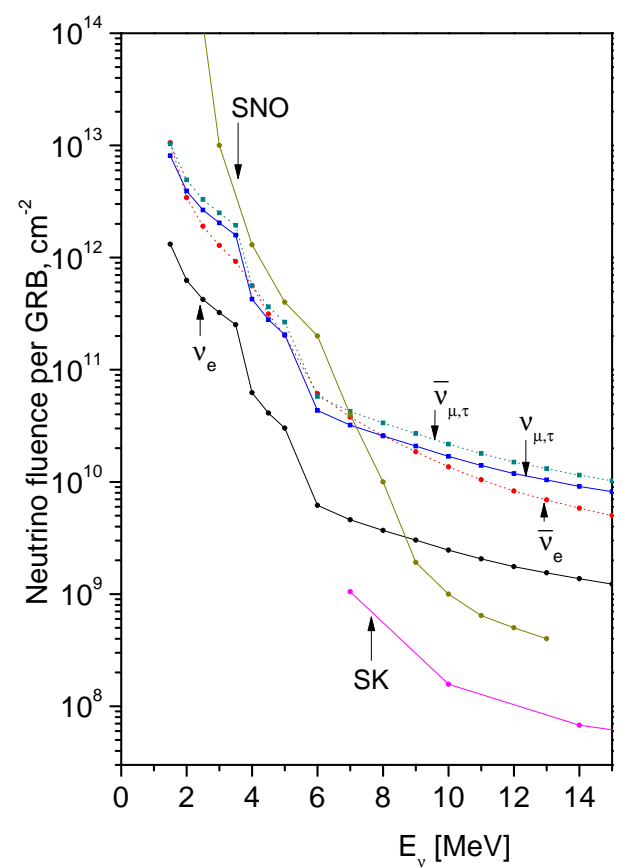

Figure 4: Borexino 90\% C.L. fluence upper limits obtained through neutrino-electron elastic scattering for $\nu_{e}$ (line 1), $\bar{\nu}_{e}(2), \nu_{\mu, \tau}(3)$, and $\bar{\nu}_{\mu, \tau}(4)$. Given are also the limits obtained for $\nu_{e}$ by SNO 20] (line 5) and SuperKamiokande [19] (line 6).

where

$$
F(T)=\int_{T^{-}}^{T^{+}} \frac{d \sigma\left(E_{\nu}, T^{\prime}\right)}{d T^{\prime}} G\left(T^{\prime}, \sigma\left(T^{\prime}\right) ; T\right) d T^{\prime} .
$$

The Gaussian function $G\left(T^{\prime}, \sigma\left(T^{\prime}\right) ; T\right)$ with variance $\sigma\left(T^{\prime}\right)^{2}$ accounts for the finite energy resolution of the detector, with $T^{-}=T-3 \sigma(T)$ and $T^{+}=T+3 \sigma(T)$. The function $F(T)$ is shown as line 4 in Fig. 3 for $E_{\nu}=14 \mathrm{MeV}$, $T_{\mathrm{th}}=1 \mathrm{MeV}$, and fluence of $1 \times 10^{10} \nu \mathrm{cm}^{-2}$ for one GRB.

The numerator in Eq. 6. $N_{90}^{\nu e}\left(E_{\nu}\right)$, is in analogy to Eq. 5, the $90 \%$ C.L. upper limit on the number of GRB-associated events per one GRB, due to neutrinos with energy $E_{\nu}$, calculated as $N_{90}^{\nu e}=(Q(0.9) \times$ $\left.\sqrt{N_{\text {in }}+N_{\text {bgr }}}\right) / N_{\text {GRB }}$, where $Q(0.9)=1.64$ is a quantile function for normal distribution. Here, $N_{\text {in }}$ and $N_{\text {bgr }}$ denote overall numbers of events in the energy interval $\left(T_{t h}, T_{\nu}^{u p}\right)$ detected in the time periods $N_{\mathrm{GRB}} \times \Delta t_{\mathrm{SIG}}$ and $N_{\mathrm{GRB}} \times \Delta t_{\mathrm{BGR}}$, respectively.

The procedure was repeated for neutrino energies $E_{\nu}$ from 1.5 to $5 \mathrm{MeV}$ in increments of $0.5 \mathrm{MeV}$ and for $E_{\nu}>$ $5 \mathrm{MeV}$ in $1.0 \mathrm{MeV}$ step. The lower integration limits $T_{t h}$ from Eq. 7 was optimized for different neutrino energies considering the shape of the spectrum decreasing with en- 
ergy (Fig. 3). The three blue lines indicated in Fig. 3 with indices 1,2 , and 3 show the $T_{\mathrm{th}}$, which was set to $1.0,3.0$, and $5.0 \mathrm{MeV}$, for neutrino energies $E_{\nu}$ from 1.5 to $3.5 \mathrm{MeV}$, 4.0 to $5.0 \mathrm{MeV}$, and 6.0 to $15.0 \mathrm{MeV}$, respectively.

In order to set the fluence limits for GRB-correlated neutrinos (antineutrinos) of electron and $(\mu+\tau)$ flavours individually, the corresponding cross section in Eq. 8 was set to $\sigma_{\nu_{e}}\left(\sigma_{\bar{\nu}_{e}}\right)$ and $\sigma_{\nu_{\mu \tau}}\left(\sigma_{\bar{\nu}_{\mu \tau}}\right)$, respectively, and calculated according to electroweak Standard Model [33. All limits were obtained in assumption that the whole neutrino flux consists of only individual flavour. The results obtained for two DAQ systems independently were found to be consistent within statistical uncertainty of our measurements and are summarized in Table 3 . Figure 4 shows Borexino limits obtained from the primary DAQ $\left(E_{\nu}<5 \mathrm{MeV}\right)$ and the FADC DAQ $\left(E_{\nu}>5 \mathrm{MeV}\right)$. Limits for $\nu_{e}$ obtained by SNO [20] and SuperKamiokande [19] are shown as well for comparison. The Borexino limits are the strongest for all neutrino species below $7 \mathrm{MeV}$. We note, that for the first time we obtain the limits for electron antineutrinos below IBD reaction threshold through their elastic scattering on electrons.

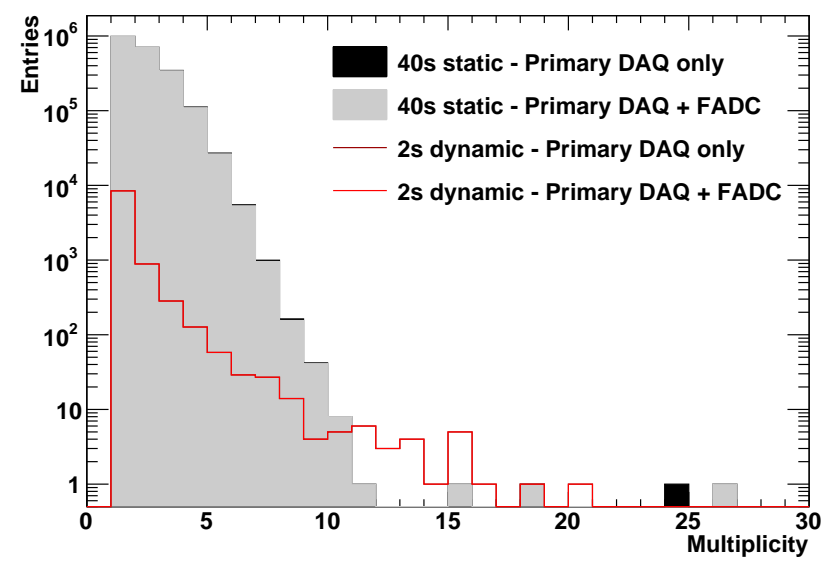

Figure 5: The distribution of multiplicities for all bursts of events in Borexino. Note that due to small differences, the results obtained without and with the use of the FADC system cannot be appreciated visually.

\subsection{Bursts of events}

We have performed a search for bursts of events in Borexino in correlation with GRBs based on 2302.0 days of data from the primary DAQ system. The FADC system was used for additional muon tagging and noise suppression for the time it was available during 1279.7 days.

To define the bursts, we have set two time windows to search for point-like events: a 40 s static and a 2 s dynamic. The $40 \mathrm{~s}$ static window has been chosen regarding the mean duration of GRBs whereas the $2 \mathrm{~s}$ dynamic one covers the maximum duration of short GRBs.

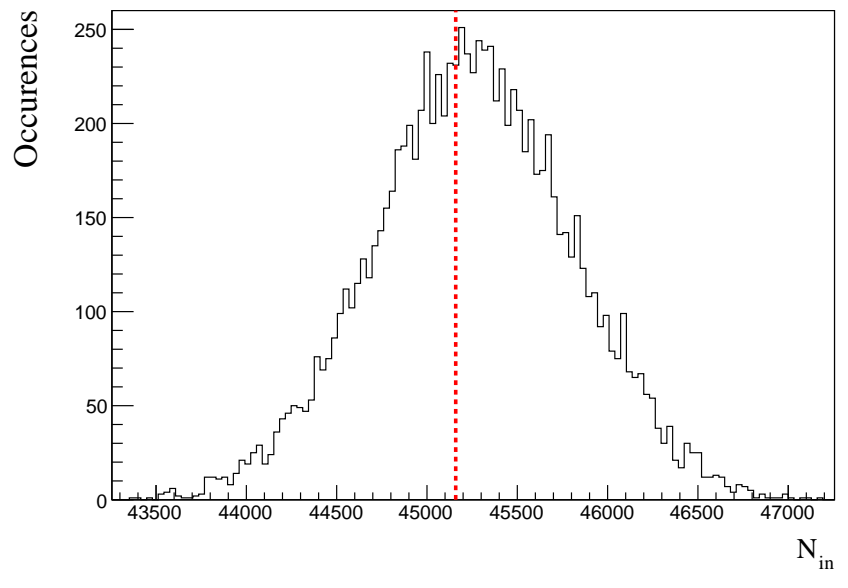

Figure 6: Distribution of the number of observed coincidences, $N_{\text {in }}$, between the observed bursts with multiplicity 3 (40 s static time window) and 10000 sets of randomly generated times, equal to the number of GRBs used in this analysis. The vertical dashed red line shows $N_{\text {in }}$ corresponding to the real GRB times.

In the static time window strategy, we have searched for non muon-correlated events with energy larger than $1 \mathrm{MeV}$. A $40 \mathrm{~s}$ time window is opened after each event and the number of such events which fall within this window is called the burst multiplicity. If one event is present in several windows, it is attributed to the one with the highest multiplicity. If one event is present in several windows with the same multiplicity, it is attributed to the first window.

The main feature of the dynamic time window strategy consists in opening a $2 \mathrm{~s}$ time window after each event with energy larger than $5 \mathrm{MeV}$. If another such event falls within this window, another $2 \mathrm{~s}$ time window is opened. This procedure continues until no more event satisfies the selection. Since in Borexino we observe about one muon every $20 \mathrm{~s}$, in this analysis we did not apply veto after muon events. To suppress cosmogenic background, the energy threshold was increased to $5 \mathrm{MeV}$.

In Fig. 5 the distributions of multiplicities from both the $40 \mathrm{~s}$ static and the $2 \mathrm{~s}$ dynamic time windows are shown. Considering the bursts with high multiplicities of $8(6)$, we have detected $10(8)$ bursts within $\Delta t_{\mathrm{SIG}}= \pm 5000 \mathrm{~s}$ time window around GRBs in the static (dynamic) time window approach. Information about bursts of highest multiplicities and in correlation with GRBs for both strategies are shown in Table 4

To show that all coincidences between bursts of events in Borexino and GRBs are accidental, simple toy MC analysis was performed. Fake GRB times, equal to the number of GRBs used in this analysis, were randomly generated 10000 times for each multiplicity separately. The results show, for both static and dynamic time windows, that the number of observed coincidences between the bursts and GRBs, $N_{\text {in }}$, is consistent with the distribution of random coincidences based on the toy MC study. This result is 
Table 3: Borexino 90\% C.L. upper limits for GRB fluences of all neutrino flavours, obtained through the study of neutrino-electron elastic scattering.

\begin{tabular}{|c|c|c|c|c|}
\hline$E_{\nu}[\mathrm{MeV}]$ & $\Phi_{\nu_{e}}\left[\mathrm{~cm}^{-2}\right]$ & $\Phi_{\bar{\nu}_{e}}\left[\mathrm{~cm}^{-2}\right]$ & $\Phi_{\nu_{\mu, \tau}}\left[\mathrm{cm}^{-2}\right]$ & $\Phi_{\bar{\nu}_{\mu, \tau}}\left[\mathrm{cm}^{-2}\right]$ \\
\hline \hline 1.5 & $1.31 \times 10^{12}$ & $1.06 \times 10^{13}$ & $8.10 \times 10^{12}$ & $1.03 \times 10^{13}$ \\
2 & $6.25 \times 10^{11}$ & $3.42 \times 10^{12}$ & $3.93 \times 10^{12}$ & $4.93 \times 10^{12}$ \\
3 & $3.23 \times 10^{11}$ & $1.28 \times 10^{12}$ & $2.03 \times 10^{12}$ & $2.50 \times 10^{12}$ \\
4 & $6.24 \times 10^{10}$ & $5.60 \times 10^{11}$ & $4.26 \times 10^{11}$ & $5.60 \times 10^{11}$ \\
6 & $6.18 \times 10^{9}$ & $6.12 \times 10^{10}$ & $4.33 \times 10^{10}$ & $5.77 \times 10^{10}$ \\
10 & $2.46 \times 10^{9}$ & $1.36 \times 10^{10}$ & $1.69 \times 10^{10}$ & $2.17 \times 10^{10}$ \\
14 & $1.37 \times 10^{9}$ & $5.82 \times 10^{9}$ & $9.12 \times 10^{9}$ & $1.15 \times 10^{10}$ \\
\hline
\end{tabular}

Table 4: Multiplicity, date, time (GMT) and duration for bursts of events in Borexino with the highest multiplicity detected within \pm 5000 s around a GRB. The last column contains names of the GRB which is closest in time. The probability that coincidences of these bursts with GRBs are accidental is $93 \%(81 \%)$ for the static (dynamic) time window.

\begin{tabular}{|c|c|c|c|}
\hline Multiplicity & Date and time (GMT) & Burst's duration, s & Closest GRB \\
\hline \multicolumn{4}{|c|}{ Static $40 \mathrm{~s}$ time window } \\
\hline 10 & 19 Dec 2010 15:45:00 & 37.1 & 101219B \\
\hline 9 & 27 Aug 2014 19:17:41 & 25.8 & $140827 \mathrm{~A}$ \\
\hline \multicolumn{4}{|c|}{ Dynamic time window } \\
\hline 15 & 19 Dec 2010 15:44:55 & 1.8 & 101219B \\
\hline 12 & 27 Aug 2014 19:17:39 & 1.9 & $140827 \mathrm{~A}$ \\
\hline
\end{tabular}

demonstrated for multiplicity 3 in Fig. 6 .

\section{Conclusions}

We have performed a search for time correlation between gamma ray bursts and events detected by Borexino and associated with neutrinos and antineutrinos reactions the inverse beta decay reaction on protons and neutrinoelectron elastic scattering. We have also searched for correlations of GRBs with short bursts of events in Borexino. The analysis was performed with data of two semiindependent data acquisition systems: the primary DAQ, optimized for events up to a few $\mathrm{MeV}$, and a Flash ADC system, designed for events above $1 \mathrm{MeV}$.

A set of 2350 GRBs observed between December 2007 and November 2015 was checked for correlations with data acquired with the primary DAQ system. A set of 1813 GRBs between December 2009 and November 2015 were also checked for correlations with the data from the FADC DAQ system. We found no statistically significant time correlations of GRBs with the events in the detector in a time windows of $\pm 5000 \mathrm{~s}$ around GRBs for $\bar{\nu}_{e}$-like events and for bursts of events, and of $\pm 1000 \mathrm{~s}$ for neutrinos of all species. The demonstrated sensitivity in the $\mathrm{MeV}$ range is close to the neutrino fluences predicted by some models of GRBs. Limits on the fluence of neutrinos of all flavours (and, separately, of electron antineutrinos) were set for neutrino energies $1.5-15 \mathrm{MeV}$. These are the most stringent bounds for GRB correlated fluence of neutrinos of all species below $7 \mathrm{MeV}$, and on the GRB correlated fluence of $\bar{\nu}_{e}$ 's in the range of $2-8 \mathrm{MeV}$.

\section{Acknowledgments}

The Borexino program is made possible by funding from INFN (Italy); the NSF (U.S.); BMBF, DFG, HGF, and MPI (Germany); RFBR: Grants No. 14-22-03031, No. 15-02-02117, and No. 16-29-13014 (Russia); NCN Poland (Grant No. UMO-2013/10/E/ST2/00180). We acknowledge the generous support and hospitality of the Laboratori Nazionali del Gran Sasso (LNGS).

\section{References}

\section{References}

[1] W.H. Lee and E. Ramirez-Ruiz, Accretion modes in collapsars: prospects for GRB production, Astrophys. J. 641 (2006) 96.

[2] P. Mészáros, Gamma-ray bursts, Rep. Prog. Phys. 69 (2006) 2259.

[3] S. Sahu and J.C. D'Olivo, Can there be neutrino oscillation in Gamma-Ray Bursts fireball?, Phys. Rev. D 71 (2005) 047303.

[4] F. Halzen and G. Jaczko, Signatures of $\gamma$ ray bursts in neutrino telescopes, Phys. Rev. D 54 (1996) 2779.

[5] P. Mészáros, Gamma Ray Bursts as Neutrino Sources, arXiv:1511.01396v1.

[6] P.A.R. Ade et al. (Planck Collaboration), Planck 2013 results. XVI. Cosmological parameters, Astron. \& Astophys., 571 (2014) A16. 
[7] V. Berezinsky, B. Hnatyk, A. Vilenkin, Gamma ray bursts from superconducting cosmic strings, Phys. Rev. D 64 (2001) 043004.

[8] K. S. Cheng, Yun-Wei Yu, T. Harko, High-Redshift GammaRay Bursts: Observational Signatures of Superconducting Cosmic Strings?, Phys. Rev. Lett. 104 (2010) 241102.

[9] M. Agostini et al. (Borexino Collaboration), Spectroscopy of geoneutrinos from 2056 days of Borexino data, Phys. Rev. D 92 (2015) 031101.

[10] P. Mészáros and E. Waxman, TeV neutrinos from successful and choked gamma-ray bursts, Phys. Rev. Lett. 87 (2001) 171102.

[11] E. Waxman, Neutrino astronomy and gamma-ray bursts, Phil. Trans. Roy. Soc. Lond. A 365 (2007) 1323.

[12] A. Achterberg et al. (IceCube Collaboration), Search for neutrino-induced cascades from gamma-ray bursts with AMANDA, Astrophys. J. 664 (2007) 397.

[13] A.G. Vieregg et al. (ANITA Collaboration), The First Limits on the Ultra-high Energy Neutrino Fluence from Gamma-Ray Bursts, Astrophys. J. 736 (2011) 50.

14] S. Adrián-Martínez et al. (ANTARES Collaboration), First search for neutrinos in correlation with gamma-ray bursts with the ANTARES neutrino telescope, JCAP 03 (2013) 006

[15] S. Adrián-Martínez et al. (ANTARES Collaboration), Search for muon neutrinos from gamma-ray bursts with the ANTARES neutrino telescope using 2008 to 2011 data, Astron. \& Astrophys. 559 (2013) A9.

[16] A. Avrorin et al. (Baikal Collaboration), Search for neutrinos from Gamma-Ray Bursts with the Baikal neutrino telescope NT200. arXiv:0910.4327

[17] R. U. Abbasi et al. (IceCube Collaboration), Search for muon neutrinos from Gamma-Ray Bursts with the IceCube neutrino telescope, Astrophys. J. 710 (2010) 346.

[18] M.G. Aartsen et al. (IceCube Collaboration), Search for Prompt Neutrino Emission from Gamma-Ray Bursts with IceCube, Astrophys. J. 805 (2015) L5.

[19] S. Fukuda et al. (Super-Kamiokande Collaboration), Search for neutrinos from Gamma-Ray Bursts using Super-Kamiokande, Astrophys. J. 578 (2002) 317.

[20] B. Aharmim et al. (SNO Collaboration), A search for astrophysical burst signals at the Sudbury Neutrino Observatory, Astropart. Phys. 55 (2014) 1.

[21] K. Asakura et al. (KamLAND Collaboration), Study of Electron Anti-neutrinos Associated with Gamma-Ray Bursts Using KamLAND, Astrophys. J. 806 (2015) 87.

[22] M.M. Kochkarov et al., Search for low-energy neutrinos from gamma-ray bursts at the Baksan Underground Scintillation Telescope, Phys. Part. Nucl. 46 (2015) 197.

[23] G. Alimonti et al. (Borexino Collaboration), The liquid handling systems for the Borexino solar neutrino detector, Nucl. Instr. and Methods A 609 (2009) 58.

[24] G. Bellini et al. (Borexino Collaboration), Final results of Borexino Phase-I on low-energy solar neutrino spectroscopy, Phys. Rev. D 89 (2014) 112007.

[25] G. Bellini et al. (Borexino Collaboration), Neutrinos from the primary proton-proton fusion process in the Sun, Nature 512 (2014) 383.

[26] G. Alimonti et al. (Borexino Collaboration), The Borexino detector at the Laboratori Nazionali del Gran Sasso, Nucl. Instr. and Methods A 600 (2009) 568.

[27] G. Bellini et al. (Borexino Collaboration), Muon and Cosmogenic Neutron Detection in Borexino, J. Instrum. 6 (2011) P05005.

[28] H. Back et al. (Borexino Collaboration), Borexino calibrations: Hardware, Methods, and Results, J. Instrum. 7 (2012) P10018.

[29] http : //grbweb.icecube.wisc.edu/

[30] G. Bellini et al. (Borexino Collaboration), Study of solar and other unknown anti-neutrino fluxes with Borexino at LNGS, Phys. Lett. B 696 (2011) 191.

[31] G.J. Feldman and R.D. Cousins, A Unified Approach to the Classical Statistical Analysis of Small Signals, Phys. Rev. D57 (1998) 3873

[32] A. Strumia, F. Vissani, Precise quasielastic neutrino/nucleon cross section, Phys. Lett. B 564 (2003) 42.

[33] L. Okun, Leptons and Quarks, 1st Edition, North Holland, 1984 